H. YANAGIHARA

KODAI MATH. J.

7 (1984), 238-247

\title{
A TAUBERIAN THEOREM FOR CERTAIN CLASS OF MEROMORPHIC FUNCTIONS
}

Dedicated to Professor M. Ozawa on the occation of his 60th birthday

\author{
By Hiroshi YanAgIHARA
}

\section{$\S 1$. Introduction.}

Let $f(z)$ be meromorphic in the plane. We define $m_{2}(r, f)$ by

$$
m_{2}(r, f)^{2}=\frac{1}{2 \pi} \int_{0}^{2 \pi}\left(\log \left|f\left(r e^{i \theta}\right)\right|\right)^{2} d \theta,
$$

and denote by $N(r, c)$ the usual Nevanlinna counting function for the $c$-points of $f$ in $|z| \leqq r$, then Miles and Shea had shown

$$
K_{2}(f) \equiv \limsup _{r \rightarrow \infty} \frac{N(r, 0)+N(r, \infty)}{m_{2}(r, f)} \geqq \frac{|\sin \pi \rho|}{\pi \rho}\left\{\frac{2}{1+\sin 2 \pi \rho / 2 \pi \rho}\right\}^{1 / 2} \equiv C(\rho)
$$

for $\rho \in\left[\mu_{*}(T(r, f)), \lambda_{*}(T(r, f))\right]$.

Further they had characterized those $f$ for which equality holds in (1) as functions which are locally Lindelöffian (or the reciprocals of such).

Let $M_{p}$ be the class of all meromorphic functions $f(z)$ of order $\rho$ defined by $g(z) / g(-z)$ with the canonical product

$$
g(z)=\prod_{n=1}^{\infty} E\left(z / a_{n}, q\right), \quad q=[\rho] .
$$

Recently by making use of Fourier series method, Ozawa proved

THEOREM A. Let $f(z)$ belongs to $M_{p}$, then

$$
\limsup _{r \rightarrow \infty} \frac{N(r, 0)}{m_{2}(r, f)} \geqq \frac{\sqrt{2}}{\sqrt{\pi \rho}} \frac{|\cos \pi \rho / 2|}{\{\pi \rho-\sin \pi \rho\}^{1 / 2}} \equiv B(\rho) .
$$

It is natural to hope that (2) holds for $\rho \in\left[\mu_{*}, \lambda_{*}\right]$ and that those $f$ for which equality holds in (2) are $f(z)=g(z) / g(-z)$ with locally Lindelöffian $g$. But when $\rho$ is an even integer, $B(\rho)>0$ and the proof is not straightforward. We need some existence lemma of strong peaks for $f \in M_{\rho}$.

We assume that the reader is familiar with the fundamental concept of Nevanlinna theory and Fourier series method developed by Miles and Shea (See

Received September 20, 1983 
W.K. Hayman [3], Miles and Shea [5], [6] and Ozawa [7]). We use the terminology from [6] without comment.

The author express his heartiest thanks to Professer M. Ozawa for his tender support in preparing this note.

\section{$\S 2$. Discussion of results.}

Our first result is following.

THEOREM 1. Let $f(z)$ be meromorphic in the plane and defined by $f(z)=$ $g(z) / g(-z)$ with an entire function $g$. Then

$$
\limsup _{r \rightarrow \infty} \frac{N(r, 0, f)}{m_{2}(r, f)} \geqq B(\rho)
$$

for $\rho \in\left[\mu_{*}(T(r, f)), \lambda_{*}(T(r, f))\right], \rho>0$.

Next we have

THEOREM 2. Under the same assumption as in theorem 1 and if

$$
\limsup _{r \rightarrow \infty} \frac{N(r, 0, f)}{m_{2}(r, f)}=B(\rho)
$$

for some $\rho \in\left[\mu_{*}(T(r, f)), \lambda_{*}(T(r, f))\right], \rho \neq$ an odd integer.

Then there exist positive sequences $r_{n} \rightarrow \infty$ and $\eta_{n} \rightarrow 0$ such that

$$
\begin{aligned}
& N(r, 0) \sim N\left(r_{n}, 0\right)\left(r / r_{n}\right)^{\prime}, \\
& N(r, 0) \sim B(\rho) m_{2}(r, f),
\end{aligned}
$$

uniformly for $r \in\left[\eta_{n} r_{n}, \eta_{n}^{-1} r_{n}\right]$ as $n \rightarrow \infty$. Further there exist $\delta_{n} \rightarrow 0$ and $\theta_{n} \in$ $[0,2 \pi)$ such that of

then

$$
S_{n}=\left\{z: \delta_{n} \leqq \arg z-\theta_{n} \leqq 2 \pi-\delta_{n}\right\},
$$

$$
N\left(r, 0 ; S_{n}\right)=o(N(r, 0, f)), \quad \eta_{n} r_{n} \leqq r \leqq \eta_{n}^{-1} r_{n}
$$

as $n \rightarrow \infty$, where $N\left(r, 0 ; S_{n}\right)$ denote the counting function for the number of zeros of $f$ in the sector $S_{n}$.

If (2.2) holds with $\rho=$ an odd integer, i.e. $N(r, 0)=o\left(m_{2}(r, f)\right)$ as $r \rightarrow \infty$, then $\rho=\mu_{*}=\lambda_{*}$ and

$$
m_{2}(r, f)=r^{o} L(r), \quad \lim _{r \rightarrow \infty} \frac{L(\sigma r)}{L(r)}=1 \quad(0<\sigma<\infty)
$$

holds.

Theorem 1 and 2 have extensions. 
THEOREM 3. Let $f(z)$ be meromorphic in the plane defined by $f(z)=g(z)$ $g\left(e^{2 \pi a} z\right)$ with an entire function $g$ and $0<a \leqq 1$. Then

$$
\limsup _{r \rightarrow \infty} \frac{N(r, 0, f)}{m_{2}(r, f)} \geqq B(a, \rho) \equiv\left\{2 \sum_{m=-\infty}^{+\infty}(1-\cos m a) \frac{4}{m^{2}-\rho^{2}}\right\}^{-1 / 2}
$$

for $\rho \in\left[\mu_{*}(T(r, f)), \lambda_{*}(T(r, f))\right], \rho \neq 0$.

THEOREM 4. Under the same assumption as in theorem 3 and if equality holds in (2.7) for some $\rho \in\left[\mu_{*}(T(r, f)), \lambda_{*}(T(r, f))\right], \mu \neq 0$ and $B(a, \rho)=0$. Then there exist sequences $r_{n} \rightarrow \infty, \eta_{n} \rightarrow 0, \delta_{n} \rightarrow 0$ and $\theta_{n} \in[0,2 \pi]$ satisfying (2.2)-(2.5). If $\rho$ satisfies $B(a, \rho)=0$ and $\rho>0$, then $\rho=\mu_{*}=\lambda_{*}$ and (2.6) holds.

Especially, if $a=1$, then we have theorem 2. Proofs of theorem 3 and 4 are quite similar as to theorem 1 and 2 . It will be done by improving the lemma 3 and be left to the reader.

Theorem 1 and 3 are not new, essentially they were proved by Ozawa ([7] theorem 4 and its extension in $\S 11$ ).

\section{$\S 3$. Preliminaries.}

To prove (2.1) we need some lemmas.

LeMma 1. Let $f(z)$ be meromorphic in the plane defined by $f(z)=g(z) / g(-z)$ with an entire function $g$. Put $a_{n}$ be zeros of $g$ and $W(z)$ by

$$
\log |f(z)|=\sum_{s<\left|a_{n}\right| \leqq R} \log \frac{\left|E\left(z / a_{n}, q\right)\right|}{\left|E\left(-z / a_{n}, q\right)\right|}+W(z)
$$

where $0<2 s \leqq|z|=r \leqq R / 2$. Then if $q \geqq 1$.

$$
\begin{aligned}
|W(z)| \leqq & V_{q}(s, r, R) \equiv A\left[(r / s)^{q_{0}-1}\left\{m_{2}(s, g)+N(2 s, 0)\right\}\right. \\
& \left.+(r / R)^{q_{0}+1}\left\{m_{2}(R, g)+N(2 R, 0)\right\}\right],
\end{aligned}
$$

where $A$ is an absolute constant and $q_{0}=2[(q+1) / 2]$; if $q=0$,

$|W(z)| \leqq V_{0}(s, r, R) \equiv A\left\{N(2 s, 0) \log (r / s)+(r / R)\left(m_{2}(R, g)+N(2 R, 0)\right)\right\}$.

Proof. According to the proof of theorem 3.b in [2],

$$
W(z)=\operatorname{Re}\left\{\sum_{m=1}^{q} d_{m}(s) z^{m}+\sum_{m=q+1}^{\infty} d_{m}(R) z^{m}+\log \prod_{\left|a_{n}\right| \leq s} \frac{\left|1-z / a_{n}\right|}{\left|1+z / a_{n}\right|}\right\}
$$

where

$$
\begin{aligned}
d_{m}(t)= & \left.\frac{1}{\pi} \int_{0}^{2 \pi} \log \left|f\left(t e^{i \theta}\right)\right| t^{-m} e^{-\imath m \theta}\right) d \theta \\
& +\underset{m t^{m}}{1} \sum_{\left|a_{n}\right| \lesssim t}\left\{\left(\bar{a}_{n} / t\right)^{m}-\left(-\bar{a}_{n} / t\right)^{m}\right\}, t \in[s, R] .
\end{aligned}
$$


Hence we have $d_{2 m}(t)=0$ and

$$
\frac{1}{4}\left|d_{2 p+1}(t)\right| \leqq \frac{\left|c_{2 p+1}(t, g)\right|}{t^{2 p+1}}+\frac{n(t, 0)}{2(2 p+1) t^{2 p+1}} .
$$

Next we have

$$
\left|\sum_{\left|a_{n}\right| \leqq s} \log \right|\left(1-z / a_{n}\right) /\left(1+z / a_{n}\right)|| \leqq 2 N(s, 0)+2 \frac{n(s, 0)}{2 q+1}(r / s)^{q} .
$$

Consequently

$$
\begin{aligned}
|W(z)| \leqq & \sum_{p=0}^{[(q+1) / 2]-1}(r / s)^{2 p+1}\left\{4 m_{2}(s, g)+2 \frac{n(s, 0)}{2 p+1}\right\} \\
& +\sum_{[(q+1) / 2]}^{\infty}(r / R)^{2 p+1}\left\{4 m_{2}(R, g)+\frac{2 n(R, 0)}{2 p+1}\right\} \\
& +2 N(s, 0)+\frac{2 n(s, 0)}{q}(r / s)^{q},
\end{aligned}
$$

and hence we have the desired result when $q \geqq 1$.

If $q=0$, we have

$$
W(z)=\operatorname{Re}\left\{\sum_{m=1}^{\infty} d_{m}(R) z^{m}+\log \underset{\left\{a_{n} \leqq s\right.}{\prod_{1 \leqslant s}}\left(1-z / a_{n}\right) /\left(1+z / a_{n}\right)\right\}
$$

and

$$
\log \prod_{\left|a_{n}\right| \leqq s}\left|\left(1-z / a_{n}\right)\left(1+z / a_{n}\right)\right| \leqq 2 N(s, 0)+n(s, 0)(1+\log r / s),
$$

and this completes the proof of lemma 1 .

LEMMA 2. Under the same assumption as in lemma 1, we have

$$
m_{2}(r, f) \leqq K_{q} r^{a_{0}+1} \int_{s}^{R} \frac{N(t, 0, g)}{t^{q_{0}}(t+r)^{2}} d t+B V_{q}(s, r, R),
$$

where $K_{q}$ and $B$ are constants depending only on $q \geqq 0$.

Proof. By lemma 1,

$$
\log |f(z)|=\log \prod_{s<\mid a_{n} \leq R}\left|E\left(z / a_{n}, q\right) / E\left(-z / a_{n}, q\right)\right|+W(z),
$$

hence we have by Minkowski's inequality,

$$
m_{2}(r, f) \leqq \sum_{s<\left|a_{n}\right| \leqq R} m_{2}\left(r /\left|a_{n}\right|, E(z, q) / E(-z, q)\right)+V_{q}(s, r, R) .
$$

For $q \geqq 1$, we have by calculating the $m$-th Fourier coefficients 


$$
m_{2}(r, G)^{2}= \begin{cases}2 \sum_{k=q_{0}}^{\infty} \frac{r^{4 k+2}}{(2 k+1)^{2}}, & r<1 \\ 2 \sum_{k=0}^{q_{0}-1}\left\{\frac{r^{4 k+2}}{(2 k+1)^{2}}-\frac{2}{(2 k+1)^{2}}\right\}+2 \sum_{k=0}^{\infty} \frac{r^{-(4 k+2)}}{(2 k+1)^{2}}, & r \geqq 1\end{cases}
$$

and if $q=0$

$$
m_{2}(r, G)^{2}= \begin{cases}\sum_{k=0}^{\infty} \frac{r^{4 k+2}}{(2 k+1)^{2}}, & r<1 \\ \sum_{k=0}^{\infty} \frac{\left.r^{-(4 k+2}\right)}{(2 k+1)^{2}}, & r \geqq 1\end{cases}
$$

where $G(z)=G(z, q) \equiv E(z, q) / E(-z, q)$ and $q_{0}=2[(q+1) / 2]$. Hence we obtain from (3.6) and (3.7)

$$
m_{2}(r, G) \leqq \begin{cases}2 r^{q_{0}+1}, & r<1 \\ 2 r^{q_{0}-1}, & r \geqq 1 .\end{cases}
$$

Thus we have from (3.5) and (3.8),

$$
\begin{aligned}
& m_{2}(r, f) \leqq 2 \int_{s}^{r}(r / t)^{q_{0}-1} d(n(t, 1 / g)-n(s, 1 / g)) \\
& \quad+2 \int_{r}^{R}(r / t)^{q_{0}+1} d(n(t, 1 / g)-n(r, 1 / g))+V_{q}(s, r, R) .
\end{aligned}
$$

Integration by parts applied twice to (3.9) yields (3.4).

LEMMA 3. Let $g$ be a entrre function and put $f$ by $f(z)=g(z) / g(-z)$. Suppose $\mu_{*}\left(m_{2}(r, f)\right)<\infty$ and $K_{2}(f)<\infty$.

If $\mu_{*}\left(m_{2}(r, f)\right)<\rho<\lambda_{*}\left(m_{2}(r, f)\right)$ and $\rho$ is not an odd integer, then there exist sequences $s_{n}, r_{n}$ and $R_{n}$ tending to $\infty$ and $\xi_{n} \rightarrow 0$ such that

$$
\begin{array}{ll}
s_{n}=o\left(r_{n}\right), \quad r_{n}=o\left(R_{n}\right) & \text { as } n \rightarrow \infty, \\
N(t, 0) \leqq N\left(r_{n}, 0\right)\left(t / r_{n}\right)^{\rho} & s_{n} \leqq t \leqq R_{n} \\
m_{2}(t, f) \leqq \xi_{n} N\left(r_{n}, 0\right)\left(s_{n} / r_{n}\right) & s_{n} \leqq t \leqq 2 s_{n} \\
m_{2}(t, f) \leqq \xi_{n} N\left(r_{n}, 0\right)\left(R_{n} / r_{n}\right) & R_{n} \leqq t \leqq 2 R_{n} .
\end{array}
$$

If $\mu_{*}\left(m_{2}(r, f)\right)=\lambda_{*}\left(m_{2}(r, f)\right)$ and $\mu_{*}\left(m_{2}(r, f)\right)$ is not an odd integer, then

$$
\liminf _{r \rightarrow \infty} \frac{N(r, 0)}{m_{2}(r, f)}>0 .
$$

Proof. We first observe that there exist sequences $s_{n}, r_{n}, R_{n}$ and $A_{n}$ tending to $\infty$ and $\delta_{n} \rightarrow 0$, such that

$$
s_{n}=o\left(r_{n}\right), \quad t_{n}=o\left(R_{n}\right) \quad \text { as } n \longrightarrow \infty
$$




$$
\begin{array}{ll}
m_{2}(t, f) \leqq m_{2}\left(t_{n}, f\right)\left(t / t_{n}\right), & s_{n} \leqq t \leqq 2 R_{n}, \\
m_{2}(t, f) \leqq \delta_{n} m_{2}\left(t_{n}, f\right)\left(t / t_{n}\right), & s_{n} \leqq t \leqq A_{n} s_{n} \quad \text { or } \quad R_{n} / A_{n} \leqq t \leqq 2 R_{n} .
\end{array}
$$

To see this, choose $\varepsilon>0$ so that $\mu_{*}<\rho-\varepsilon, \rho+\varepsilon<\lambda_{*}$, then there exist $x_{n}, y_{n}$ and $A_{n}$ tending to $\infty$ and $\gamma_{n} \rightarrow 0$ such that

$$
\begin{array}{ll}
m_{2}(t, f) \leqq\left(1+\gamma_{n}\right) m_{2}\left(x_{n}, f\right)\left(t_{n} / x_{n}\right)^{\rho+\varepsilon}, & A_{n}^{-2} x_{n} \leqq t \leqq A_{n}^{2} x_{n} \\
m_{2}(t, f) \leqq\left(1+\gamma_{n}\right) m_{2}\left(y_{n}, f\right)\left(t / y_{n}\right)^{\rho-\varepsilon} & A_{n}^{-2} y_{n} \leqq t \leqq 2 A_{n}^{2} y_{n} .
\end{array}
$$

And we may assume $A_{n}^{2} x_{n}<A_{n}^{-2} y_{n}$. Choose $t_{n} \in\left[A_{n}^{-1} x_{n}, A_{n} y_{n}\right]$ so that

$$
m_{2}\left(t_{n}, f\right) t_{n}^{-\rho} \geqq m_{2}(t, f) t^{-\rho} \quad A_{n}^{-1} x_{n} \leqq t \leqq A_{n} y_{n} .
$$

Then

$$
\begin{array}{rlrl}
m_{2}(t, f) & <\left(1+\gamma_{n}\right)\left(t / x_{n}\right)^{\varepsilon}\left(t / x_{n}\right)^{\rho}\left(x_{n} / t_{n}\right)^{\rho} m_{2}\left(t_{n}, f\right) & \\
& \leqq \delta_{n}\left(t / t_{n}\right)^{\rho} m_{2}\left(t_{n}, f\right), & A_{n}^{-2} x_{n} \leqq t \leqq A_{n}^{-1} x_{n}, \\
& A_{n} y_{n} \leqq t \leqq 2 A_{n}^{2} y_{n} .
\end{array}
$$

Thus (3.13) and (3.14) hold with $s_{n}=A_{n}^{-2} x_{n}$ and $R_{n}=A_{n}^{2} y_{n}$.

Choose $r_{n} \in\left[s_{n}, 2 R_{n}\right]$ so that

$$
N\left(r_{n}, 0\right) r_{n}^{-\rho} \geqq N(t, 0) t^{-\rho}, \quad s_{n} \leqq t \leqq 2 R_{n} .
$$

By lemma 2 and $K_{2}(f)<\infty$

$$
m_{2}\left(t_{n}, f\right) \leqq K_{q} t_{n}^{q_{0}+1} \int_{s_{n}}^{R_{n}} \frac{N(t, 0)}{t^{q_{0}}\left(t_{n}+t\right)^{2}} d t+o\left(m_{2}\left(t_{n}, f\right)\right) .
$$

Hence

$$
(1+o(1)) m_{2}\left(t_{n}, f\right) \leqq K_{q} N\left(r_{n}, 0\right)\left(t_{n} / r_{n}\right)^{\rho} \int_{0}^{\infty} \frac{u^{\rho}}{u^{q_{0}}(u+1)^{2}} d u
$$

Since $\left|\rho-q_{0}\right|<1$, the integral is convergent. We have

$$
m_{2}\left(t_{n}, f\right) \leqq(1+o(1)) \hat{K}_{\rho} N\left(r_{n}, 0\right)\left(t_{n} / r_{n}\right)^{\rho} \quad \text { as } n \rightarrow \infty .
$$

Thus for $n$ large enough,

$$
\begin{aligned}
m_{2}(t, f) \leqq \delta_{n} m_{2}\left(t_{n}, f\right)\left(t / t_{n}\right)^{\rho} \leqq 2 \hat{K}_{\rho} \delta_{n} N\left(r_{n}, 0\right)\left(t / r_{n}\right)^{\rho}, \\
s_{n} \leqq t \leqq A_{n} s_{n}, \quad A_{n}^{-1} R_{n} \leqq t \leqq 2 R_{n} .
\end{aligned}
$$

Next we note from (3.14), (3.16) and $K_{2}(f)<\infty$ that

$$
r_{n} \in\left[A_{n} s_{n}, A_{n}^{-1} R_{n}\right] \quad\left(n \geqq n_{0}\right),
$$

and (3.10) follows. 
To prove last assertion of lemma 3 suppose first that for any $\sigma>1$, there exist a sequence $\tau_{k} \rightarrow \infty$ such that $\tau_{k}=N\left(\sigma t_{k}\right) / m_{2}\left(t_{k}, f\right) \rightarrow 0$. Let $q_{0}=2[(\rho+1) / 2]$, and use

$$
m_{2}(r, f) \leqq K_{q_{0}} \int_{0}^{\infty} \frac{r^{q_{0}+1} N(t, 0)}{t^{q_{0}}(t+r)^{2}} d t
$$

where $K_{q_{0}}$ is a constant depending only on $q_{0}$.

Since $\mu_{*}\left(m_{2}\right)=\lambda_{*}\left(m_{2}\right)=\rho$, given $\varepsilon>0$ their exist $A=A(\varepsilon)$ and $x_{0}=x_{0}(\varepsilon)$ such that for any $x \geqq x_{0}$, there is a peak $y \in[x, A x]$ :

$$
\begin{array}{ll}
m_{2}(t, f) \leqq m_{2}(y, f)(t / y)^{\rho-\varepsilon} & \left(x_{0} \leqq t \leqq y\right) . \\
m_{2}(t, f) \leqq m_{2}(y, f)(t / y)^{\rho+\varepsilon} & (y \leqq t<\infty) .
\end{array}
$$

(See [1], p. 410 and [6], p. 178). Choose $\varepsilon>0$ so that $\rho+\varepsilon<q_{0}+1$ and $q_{0}-1$ $<\rho-\varepsilon$. Then for all large $k$ there exist peaks $y_{k} \in\left[t_{k} / A, t_{k}\right]$; if $s_{k} \in\left(x_{0}, y_{k}\right)$,

$$
\begin{aligned}
m_{2}\left(y_{k}, f\right) \leqq & \left\{B K_{2}(f) \int_{x_{0}}^{s_{k}} m_{2}(t, f)+\int_{s_{k}}^{\sigma t_{k}} N\left(\sigma t_{k}, 0\right)\right. \\
& \left.+B K_{2}(f) \int_{\sigma t_{k}}^{\infty} m_{2}(t, f)\right\} \frac{y_{k}^{q_{0}+1}}{t^{q_{0}}\left(t+y_{k}\right)^{2}} d t+O\left(y_{k}^{q_{0}-1}\right) \\
1 \leqq & B K_{2}(f) \int_{y_{k} / s_{k}}^{y_{k} / x_{0}} \frac{u^{q_{0}-\rho+\varepsilon}}{(1+u)^{2}} d u+\tau_{k} A^{\rho+\varepsilon} \int_{y_{k} / \sigma t_{k}}^{y_{k} / s_{k}} \frac{u^{q_{0}}}{(1+u)^{2}}-d u \\
+ & B K_{2}(f) \int_{0}^{y_{k} / \sigma t_{k}} \frac{u^{q_{0}-\rho-\varepsilon}}{(1+u)^{2}} d u+o(1) .
\end{aligned}
$$

We determine the $s_{k}$ so that $s_{k} \rightarrow \infty, y_{k} / s_{k} \rightarrow \infty$ and

$$
\tau_{k} \int_{0}^{y_{k} / s_{k}} \frac{u^{q_{0}}}{(1+u)^{2}} d u \rightarrow 0
$$

Then since $y_{k} \leqq t_{k} \leqq A y_{k}$,

$$
1 \leqq B K_{2}(f) \int_{0}^{1 / \sigma} \frac{u^{q_{0}-\rho-\varepsilon}}{(1+u)^{2}} d u+o(1) \quad \text { as } \quad k \rightarrow \infty,
$$

a contradiction if $\sigma$ has been chosen large enough. Thus $m_{2}(r, f) \leqq C_{1} N(\sigma r, 0)$. Since $K_{2}(f)<\infty$, we have $\mu_{*}(N)=\mu_{*}\left(m_{2}\right)=\lambda_{*}(N)=\lambda_{*}\left(m_{2}\right)=\rho_{\text {. }}$ In particular $N(\sigma r, 0) \leqq C_{2} N(r, 0)$, and we have

$$
\liminf _{r \rightarrow \infty} \frac{N(r, 0)}{m_{2}(r, f)}>0 .
$$

\section{$\S 4$. Proof of Theorems 1 and 2 .}

We may assume $K_{2}(f)<\infty$, since otherwise (2.1) is trivial. Hence we have $\mu_{*}\left(m_{2}\right)=\mu_{*}(T)$ and $\lambda_{*}\left(m_{2}\right)=\lambda_{*}(T)$. 
Let $\rho \in\left[\mu_{*}, \lambda_{*}\right](\rho>0)$ be not odd and choose $a=a(\rho) \Subset\left(0, e^{-1}\right)$ by

$$
1-\log a>\rho^{-1}, \quad(a e)^{\rho}(1-\log a)<1 .
$$

Let $q_{0}=2[(\rho+1) / 2]$ and put $f_{n}(z)$ by

$$
f_{n}(z)=\prod_{s_{n}<\left|a_{n}\right| \leq a R_{n}} E\left(z / a_{n}, q^{0}\right) / E\left(-z / a_{n}, q_{0}\right)
$$

where $s_{n}, r_{n}$ and $R_{n}$ satisfy (3.10), (3.12) and $\gamma_{n} \rightarrow 0$

$$
N(t, 0) \leqq\left(1+\gamma_{n}\right) N\left(r_{n}, 0\right)\left(t / r_{n}\right)^{\rho}, \quad s_{n} \leqq t \leqq R_{n} .
$$

Define associated functions $G_{n}(z)$ and $F_{n}(z)$ by

$$
\begin{gathered}
G_{n}(z)=\prod_{s_{n}<\left|a_{n}\right| \leqq a R_{n}} E\left(z /\left|a_{n}\right|, q_{0}\right), \\
F_{n}(z)=G_{n}(z) / G_{n}(-z) .
\end{gathered}
$$

Put $N_{n}(t, 0)=N\left(t, 1 / F_{n}\right)$ so that by (3.12) and (4.2)

$$
\begin{gathered}
N_{n}\left(r_{n}, 0\right) \sim N\left(r_{n}, 0\right) \quad \text { as } \quad n \rightarrow \infty . \\
N_{n}(t, 0) \leqq\left(1+\gamma_{n}\right) N\left(r_{n}^{\prime}, 0\right)\left(t^{\prime} r_{n}\right)^{n}, \quad 0<t<\infty .
\end{gathered}
$$

We apply lemma 1 on $|z|=r_{n}$, and obtain

$$
\log |f(z)|=\log \left|f_{h}(z)\right|+o\left(N\left(r_{n}, 0\right)\right), \quad \text { as } n \rightarrow \infty .
$$

Since $m_{2}(r, f) \leqq m_{2}\left(r, F_{n}\right)$, we have

$$
m_{2}\left(r_{n}, f\right) \leqq(1+o(1)) m_{2}\left(r_{n}, F_{n}\right), \quad \text { as } n \rightarrow \infty .
$$

Let

$$
L_{n}(z)=\prod_{k=1}^{\infty} E\left(z / d_{k}, q_{0}\right) / E\left(-z / d_{k}, q_{0}\right)
$$

be the meromorphic function with positive zeros $d_{k}$ and negative poles $-d_{k}$ satisfying

$$
n(t, 0)=\left[\rho\left(t / r_{n}\right)^{\rho} N\left(r_{n}, 0\right)\right] \quad 0<t<\infty .
$$

Then for each $n \geqq 1$

$$
\begin{array}{ll}
N\left(t, 1 / L_{n}\right) \leqq\left(t / r_{n}\right)^{\rho} N\left(r_{n}, 0\right) & 0<t<\infty, \\
N\left(t, 1 / L_{n}\right) \sim\left(t / r_{n}\right)^{\rho} N\left(r_{n}, 0\right) & \text { as } t \rightarrow \infty .
\end{array}
$$

and

$$
\begin{gathered}
c_{2 p}\left(r_{n}, L_{n}\right)=0 \\
\left|c_{2 p+1}\left(r_{n}, L_{n}\right)\right| \sim N\left(r_{n}, 0\right) \frac{\rho^{2}}{\left|(2 p+1)^{2}-\rho^{2}\right|} \quad \text { as } n \rightarrow \infty,
\end{gathered}
$$


uniformly in $p$. Hence

$$
\left|c_{m}\left(r_{n}, F_{n}\right)\right| \leqq(1+o(1))\left|c_{m}\left(r_{n}, L_{n}\right)\right| \quad \text { as } n \rightarrow \infty,
$$

uniformly in $m$. We deduce

$$
m_{2}\left(r_{n}, F_{n}\right) \leqq(1+o(1)) m_{2}\left(r_{n}, L_{n}\right)=(1+o(1)) N\left(r_{n}, 0\right) B(\rho)^{-1}
$$

and thus (2.1) follows (See [4] p. 185).

Proof of theorem 2. Let $\rho>0$ satisfy (2.2) and be not odd. Then by the proof of theorem 1 there exist meromorphic $f_{n}$ and associated $G_{n}, F_{n}$, and $L_{n}$ satisfying (3.10), (3.12) and (4.2). Let $M>1$ be large and suppose that there exist $x_{n} \in\left[r_{n}, M r_{n}\right]$ and $\sigma \in(0,1)$ such that

Then

$$
N\left(x_{n}, 0\right)<\sigma^{2} N\left(r_{n}, 0\right)\left(x_{n} / r_{n}\right)^{\rho} \quad \text { for infinitely many } n .
$$

$$
\limsup _{n \rightarrow \infty} \frac{\left|c_{q_{0}+1}\left(r_{n}, L_{n}\right)\right|}{\left|c_{q_{0}+1}\left(r_{n}, F_{n}\right)\right|}>1,
$$

a contradiction. We conclude

$$
N(x, 0)=(1+o(1)) N\left(r_{n}, 0\right)\left(x / r_{n}\right)^{o}, \quad r_{n} \leqq x \leqq M r_{n},
$$

uniformly as $n \rightarrow \infty$. Thus by lemma 1 and lemma 3

$$
\log |f(z)|=\log \left|f_{n}(z)\right|+o\left(m_{2}(r, f)\right), \quad r_{n} \leqq|z|=r \leqq M r_{n},
$$

uniformly as $n \rightarrow \infty$ and we have

$$
m_{2}(r, f) \leqq(1+o(1)) m_{2}\left(r, F_{n}\right) \leqq(1+o(1)) B(\rho)^{-1} N(r, 0)
$$

uniformly on $r_{n} \leqq|z|=r<M r_{n}$ as $n \rightarrow \infty$; by (2.2) equality holds throughout in (4.10).

Now by (4.8) there exist $\varepsilon_{n}$ tending to 0 with

$$
\left|c_{m}\left(r_{n}, f_{n}\right)\right|>\left(1-\varepsilon_{n}\right)\left|c_{m}\left(r_{n}, F_{n}\right)\right|
$$

for $m=q_{0}+1, q_{0}+3$. By lemma (2.2) of [6], there exist $\delta_{n} \rightarrow 0$ and $\phi_{n}, \phi_{n} \in$ $[0,2 \pi)$ such that if

$$
\begin{array}{ll}
\hat{I}_{n}=\bigcap_{\jmath=0}^{q_{0}} S\left(\phi_{n}+2 \jmath \pi /\left(q_{0}+1\right), \delta_{n}\right) & \tilde{I}_{n}=\bigcap_{\jmath=0}^{q_{0}+2} S\left(\phi_{n}+2 j \pi /\left(q_{0}+3\right), \delta_{n}\right) \\
\hat{G}_{n}(z)=\prod_{\substack{s_{n}<a_{\nu} \mid \leq a R_{n} \\
a_{\nu} \in \hat{I}_{n}}} E\left(z / a_{n}, q_{0}\right) \quad \tilde{G}_{n}(z)=\prod_{\substack{s_{n}<\left|a_{\nu}\right| \leq a R_{n} \\
a_{\nu} \in \tilde{I}_{n}}} E\left(z / a_{n}, q_{0}\right)
\end{array}
$$

and put $\hat{F}_{n}(z)=\hat{G}_{n}(z) / \hat{G}_{n}(-z)$ and $\tilde{F}_{n}(z)=\tilde{G}_{n}(z) / \tilde{G}_{n}(-z)$, then

$$
\begin{aligned}
& \left|c_{q_{0}+1}\left(r_{n}, F_{n}\right)\right|<\sqrt{\varepsilon_{n}}\left|c_{q_{0}+1}\left(r_{n}, F_{n}\right)\right|, \\
& \left|c_{q_{0}+3}\left(r_{n}, F_{n}\right)\right|<\sqrt{\varepsilon_{n}}\left|c_{q_{0}+1}\left(r_{n}, F_{n}\right)\right| .
\end{aligned}
$$


One consequence of $(4.14)$ is

$$
N\left(t, 0, F_{n}\right)+N\left(t, 0, F_{n}\right) \leqq M^{-n / 8} N\left(t, 0, L_{n}\right) \quad M^{3 / 1 / r_{n}} \leqq t \leqq M r_{n} .
$$

And (4.15) shows (2.5). (See [6] p. 183).

If (2.2) holds for $\rho=a$ positive odd integer, the proof of (2.6) is quite similar to (14) of [6] and will be left to the reader.

\section{REFERENCES}

[1] D. DKasin and D.F. Shea, Polya peaks and the oscillation of positive functions, Proc. Amer. Math. Soc., 34 (1972), 403-411.

[2] A. Edrei AND W.H.J. Fuchs, On the growth of meromorphic functions with several deficient values, Trans. Amer. Math. Soc., 93 (1959), 292-328.

[3] W.K. Hayman, Meromorphic functions, Clarendon Press, Oxford, 1964.

[4] M. Herns, Complex function theory, Academic Press, New York and London (1968).

[5] J. Miles And D.F. Shea, An extremal problem in value-distribution theory, Quart. J. Math. (2) 24 (1973), 377-383.

[6] J. Miles And D.F. Shea, On the growth of meromorphic functions having at least one deficient value, Duke Math. J., 43 (1976), 171-186.

[.] M. OzAWA, On the growth of meromorphic functions, to appear.

Department of Mathematics

TOKyo Institute of TECHNOLOGY

Oh-okayama, Meguro-kU, Tokyo, Japan 